\title{
Comparison of the diagnostic accuracy of lymphatic endothelium markers: Bayesian approach
}

\author{
Evangelos Evangelou ${ }^{1}$, Panayiotis A Kyzas ${ }^{1}$ and Thomas A Trikalinos ${ }^{1,2}$ \\ ${ }^{1}$ Department of Hygiene and Epidemiology, University of Ioannina School of Medicine, Ioannina, Greece \\ and ${ }^{2}$ Institute for Clinical Research and Health Policy Studies, Tufts-New England Medical Center, Boston, \\ MA, USA
}

\begin{abstract}
Tumor lymphatic density is evaluated by means of specific lymphatic endothelium markers, and is a potential predictor of clinically meaningful outcomes. There are many claims on the postulated superiority of some of these markers to identify lymphatics, always in the absence of quantitative data. We therefore compared the diagnostic accuracy of the antibody against podoplanin and the commercially available D2-40, employing Bayesian statistics to account for the absence of a gold standard. We used the pan-endothelial marker CD34 to identify 23542 distinct blood and lymphatic vessels in sections from 30 formalin-fixed, paraffin-embedded archival tissue blocks of head and neck squamous cell carcinoma specimens. We stained two adjacent sections with podoplanin and D2-40 and identified the continuum of each stained vessel in the sections with a comprehensive method. Overall, 1864 vessels were stained with both markers, 119 only with podoplanin and 391 only with D2-40. Significantly more vessels with intraluminal red blood cells were stained with D2-40 compared to podoplanin (McNemar's $\boldsymbol{P}<\mathbf{0 . 0 0 0 1 )}$. Both antibodies had extremely high specificity $(99.7 \%(95 \%$ credible interval (Crl): $99.5-99.9 \%)$ and $98.8 \%$ (95\% Crl: 98.3-99.5\%) for podoplanin and D2-40, respectively) and very high sensitivity (92.6\% (95\% Crl: 86.1-97.9\%) and 97.3\% (95\% Crl: 94.9-99.2\%) for podoplanin and D2-40, respectively). Inferences were qualitatively similar when we took into account in the analyses the possibility that the two tests (antibodies) may be correlated. We calculated that $96.3 \%$ (95\% Crl: $94.2-98.6 \%)$ of the vessels stained with podoplanin and $88.9 \%$ (95\% Crl: 83.9-95.7\%) of the vessels stained with D2-40 were truly lymphatics. These numbers were in agreement with the observed number of stained vessels without intraluminal red blood cells. Our results suggest that both antibodies are excellent lymphatic endothelium markers and that there may be little reason to prefer either of them in most settings.
\end{abstract}

Modern Pathology (2005) 18, 1490-1497. doi:10.1038/modpathol.3800457; published online 1 July 2005

Keywords: podoplanin; D2-40; specificity; Bayesian inference; lymphangiogenesis

The mechanisms for the metastasis of epithelial neoplasms via lymphatic vessels have recently become the focus of intensive research activity. ${ }^{1-5}$ Researchers probe into the biology of lymphatics, the interactions of tumor cells with the lymphatic vessels, and the mechanisms of lymphangiogenesis. Central to all these efforts was the discovery of lymphatic endothelium markers, such as LYVE-1, the antibody against podoplanin, D2-40, prox-1 and VEGFR-3. ${ }^{1,5}$ It has been postulated that the density of the lymphatic vessels is a predictor of clinically

Correspondence: Dr PA Kyzas, DDS, Department of Hygiene and Epidemiology, University of Ioannina School of Medical, Ioannina 45 110, Greece.

E-mail: md03791@cc.uoi.gr

Received 11 April 2005; revised 31 May 2005; accepted 1 June 2005; published online 1 July 2005 meaningful outcomes in a number of malignancies. $^{6-13}$ One of the most critical steps in the assessment of lymphatic vessel density is the selection of the optimal lymphatic endothelium marker, ${ }^{1,2,5,12-23}$ in order to avoid spurious findings arising from the low accuracy of the staining. However, given the absence of a gold standard, the evaluation of the sensitivity and the specificity of the lymphatic endothelium markers is a daunting task.

Antibody against podoplanin is considered by many researchers to be the most specific lymphatic endothelium marker available. ${ }^{1,5,9,14,15,17,21}$ Such claims are not based on formal quantitative comparisons between different lymphatic endothelium markers in the same samples, despite the numerous relative calls in the literature..$^{3,12,16,20-22}$ We set out to compare the diagnostic accuracy of podoplanin and 
D2-40 in the same samples, employing a Bayesian approach to account for the absence of a gold standard.

\section{Materials and methods}

\section{Tissue Samples}

We used 4- $\mu$ m-thick sections from a sample of 30 formalin-fixed, paraffin-embedded archival tissue blocks of head and neck squamous cell carcinoma specimens. In all, 25 of these were biopsy specimens, while five were taken from excised tumors. We counted the total number of vessels (both blood and lymphatic) using the pan-endothelial marker CD34. ${ }^{24,25}$ A monoclonal antibody raised against podoplanin, as described previously ${ }^{26,27}$ (kindly provided by Dr Geleff, Department of Pathology, University of Vienna, Austria), and the commercial monoclonal antibody D2-40 (SIG-730, Signet Laboratories, Dedham, MA, USA, dilution 1:40) were used to stain lymphatic vessels in two adjacent sections. Immunohistochemistry was performed using the EnVision System (DAKO, Copenhagen, Denmark), as previously described. ${ }^{13}$

\section{Evaluation of Staining Patterns}

We assessed the expression of the antibody against podoplanin and D2-40 in each section using an Olympus BX-51 microscope at $\times 400$ magnification. We aimed to identify whether each single vessel in any section was stained with both antibodies, only with podoplanin or only with D2-40. For this reason, we recognized the continuity of each vessel in the adjacent sections. We decided on a set of $a$ priori formulated rules to evaluate the staining. First, we specified that vessels containing red blood cells were most likely blood vessels. ${ }^{28}$ We therefore considered all stained vessels with intraluminal erythrocytes as potential 'false-positives'. We recorded these potential 'false-positives' in order to check the plausibility of our calculations. Second, we considered vessels, which appeared joined in one section and separated in the other, as one in both sections. Third, we excluded any vessels that were identified only in one section (stained by either antibody) but could not be identified in the adjacent section. Hence, we only counted vessels that could be identified in both sections stained or unstained by the lymphatic markers. Finally, to ensure that no double counting occurred, we photographed areas with high lymphatic vessel density and compared them in the neighboring sections with image processing software. Counts of positively stained vessels with either antibody, as well as potential 'false-positive' counts were tabulated in $2 \times 2$ contingency tables. We compared whether the two antibodies had any difference in the number of identified lymphatic vessels and in their potential 'false-positive' counts using McNemar's test.

\section{Assessment of Diagnostic Accuracy}

The two antibodies may be conceived as two diagnostic tests to identify lymphatics. In order to assess their accuracy, one has to calculate their sensitivity (the proportion of positively stained vessels that are indeed lymphatic vessels) and their specificity (the proportion of the unstained vessels that are not lymphatic vessels). ${ }^{29}$ In the absence of a gold standard, it is not possible to use 'classical', frequentist approaches to estimate these quantities. ${ }^{30}$ In simple terms, there are five unknown parameters (the sensitivities and the specificities of the two antibodies as well as the true prevalence of the lymphatic vessels in the examined sections), which cannot be estimated directly from the data. However, such problems can be addressed with nonfrequentist, Bayesian approaches, using information on staining combinations with both antibodies, as previously proposed. ${ }^{30}$ The basic idea behind any Bayesian assessment of diagnostic accuracy is to utilize external information in order to bypass the restrictions posed by the unknown quantities. Prior knowledge (knowledge not stemming from the data) can be used to describe the unknown quantities mathematically, as distributions of probable values. The available data from the current study are summarized in the table of positively stained vessels with either antibody; the potential 'false-positive' counts have not been taken into account in the assessment of the diagnostic accuracy. Finally, the information conveyed by the data is combined with the prior information using Bayes' theorem, to yield the final, 'posterior' knowledge on the specificities and sensitivities of the two antibodies. One can then easily draw inferences on the diagnostic accuracy of the two tests.

The first step in any Bayesian analysis is to obtain reasonable prior distributions, based on sound judgments. In our case, a priori very little was known about the true number of the lymphatic vessels in the sections. We approximated this uncertainty by using a uniform prior distribution for the prevalence of lymphatics (the proportion of true lymphatic vessels among all vessels) in the sections. This distribution considers all prevalence values between 0 and $100 \%$ as equally probable, and thus is completely noninformative. Limited information was also available on the sensitivity of each antibody. We assigned a broad range of probable values between 60 and $100 \%$ for the sensitivities of both antibodies (Figure 1). This was based on observations from electron microscopy studies, ${ }^{26}$ where all small lymphatic vessels were intensely stained with podoplanin and larger lymphatics were not intensely stained. Since the vast majority of the lymphatic vessels are small, this prior seems to be 
1492

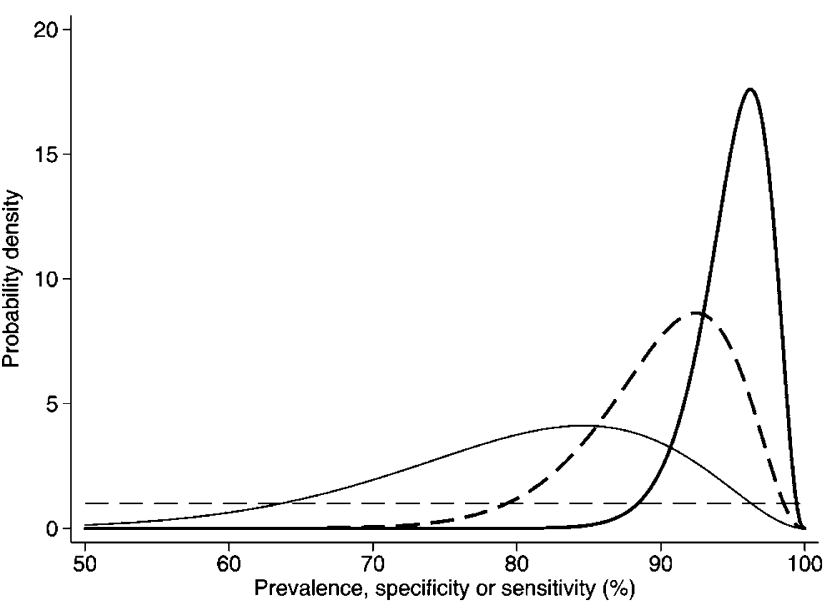

Figure 1 Prior distributions for the unknown quantities. Bayesian approaches borrow strength from prior information (information not stemming from the data) on the five unknown parameters in the problem. Prior information is expressed as distributions of probable values. The same $\operatorname{Beta}(12,3)$ prior was set for the sensitivities of the two tests (thin solid line). A Beta(71.25,3.75) prior was used for the specificity of the antibody against podoplanin (thick solid line) and a Beta(31.5,3.5) for the specificity of D2-40 (thick dashed line). The prior for the prevalence (thin dashed line) was a uniform distribution. All distributions extend from 0 to $100 \%$. Narrow distributions imply that a narrow range of values is more probable (has a higher probability density).

valid. We specified the same prior for D2-40. These monoclonal antibodies are considered to be highly specific. ${ }^{1,5,9,14,15,17,21,26}$ Therefore, we assigned to both antibodies high specificities. Also, prior beliefs regarded the antibody against podoplanin as the most specific lymphatic endothelium marker; ${ }^{1,5,9,14,15,17,21}$ hence, we set priors between 90 and $100 \%$ for the specificity of the antibody against podoplanin, and between 80 and $100 \%$ for D2-40 (Figure 1). In secondary analyses, we examined if the results changed when we assigned the same priors between 80 and $100 \%$ to the specificity of both antibodies. Various other vague (noninformative) prior distributions were also used. A first set of analyses considered the two tests (antibodies) statistically independent. This assumption may not hold if D2-40 recognizes podoplanin protein. ${ }^{31}$ This would mean that the two tests are correlated (not statistically independent), and should be taken into account mathematically. ${ }^{32,33}$ Thus, in a second set of analyses, we allowed for possible correlation between the two tests. We specified a prior for this correlation that ranged between negligible and very high. (Details on the technicalities of the approaches, as well as the results of sensitivity analyses, are provided as online supplementary information.)

Based on the estimates of diagnostic accuracy, we calculated how many of the positively stained vessels would truly be lymphatics in different tissues, where lymphatics may be commonly or sparsely found. This proportion is otherwise called the positive predictive value of each antibody and is dependent on the prevalence of the lymphatics in a section. Finally, we compared the predicted number of false positively stained vessels in our samples with the number of stained vessels with intraluminal erythrocytes in order to assess the plausibility of our findings. Given that the counts of the potential 'false-positive vessels' were not taken into account in the estimation of the diagnostic accuracies, this may serve as an external validation of the calculations.

Analyses were conducted in R 2.0.1 (R Foundation for Statistical Computing, Vienna), JAGS 0.80 (Martyn Plummer, 2005) and Intercooled Stata 8.2 (Stata Corp., College Station, TX, USA). Results are presented in the form of posterior medians and 95\% equally tailed posterior credible intervals (CrI) (95\% CrI). CrIs are analogues of the usually encountered (frequentist) $95 \%$ confidence intervals. McNemar test $P$-values are two tailed, and are considered significant for $P<0.05$.

\section{Results}

\section{Immunostaining}

Typical staining patterns with both antibodies for lymphatic vessels are shown in Figure 2. Generally, positively stained vessels with either lymphatic marker were thin walled and irregularly shaped, and were often comprised by two to three endothelial cells. Overall, we identified 23542 different blood vessels and lymphatics in the 30 specimens we examined. Of these, 1983 and 2255 were stained with the antibody against podoplanin and with D2-40, respectively (Table 1 ). The distribution of the vessel counts in the $2 \times 2$ tables across the 30 specimens did not differ (Breslow-Day $P=1.00$ ). Significantly more vessels were positive for D2-40 $(P<0.001)$. There were 394 vessels that were positive either for podoplanin or D2-40, but had intraluminal red blood cells and were considered as potential 'false-positives' (Table 2). These potential 'false-positives' were significantly more common with D2-40 compared to podoplanin $(P<0.001)$.

\section{Diagnostic Accuracy}

Under the assumption that the two tests are statistically independent, both antibodies were found to be extremely specific for the identification of lymphatic vessels. The posterior estimates of the specificities of the two antibodies were clearly separated (Figure 3a), although their absolute difference was very small. Specificities were 99.7\% (95\% CrI: 99.5-99.9\%) and 98.8\% (95\% CrI: 98.3$99.5 \%$ ) for the antibody against podoplanin and D2-40, respectively. Podoplanin had lower sensitivity $(92.6 \%$ (95\% CrI: $86.1-97.9 \%)$ ) compared to 

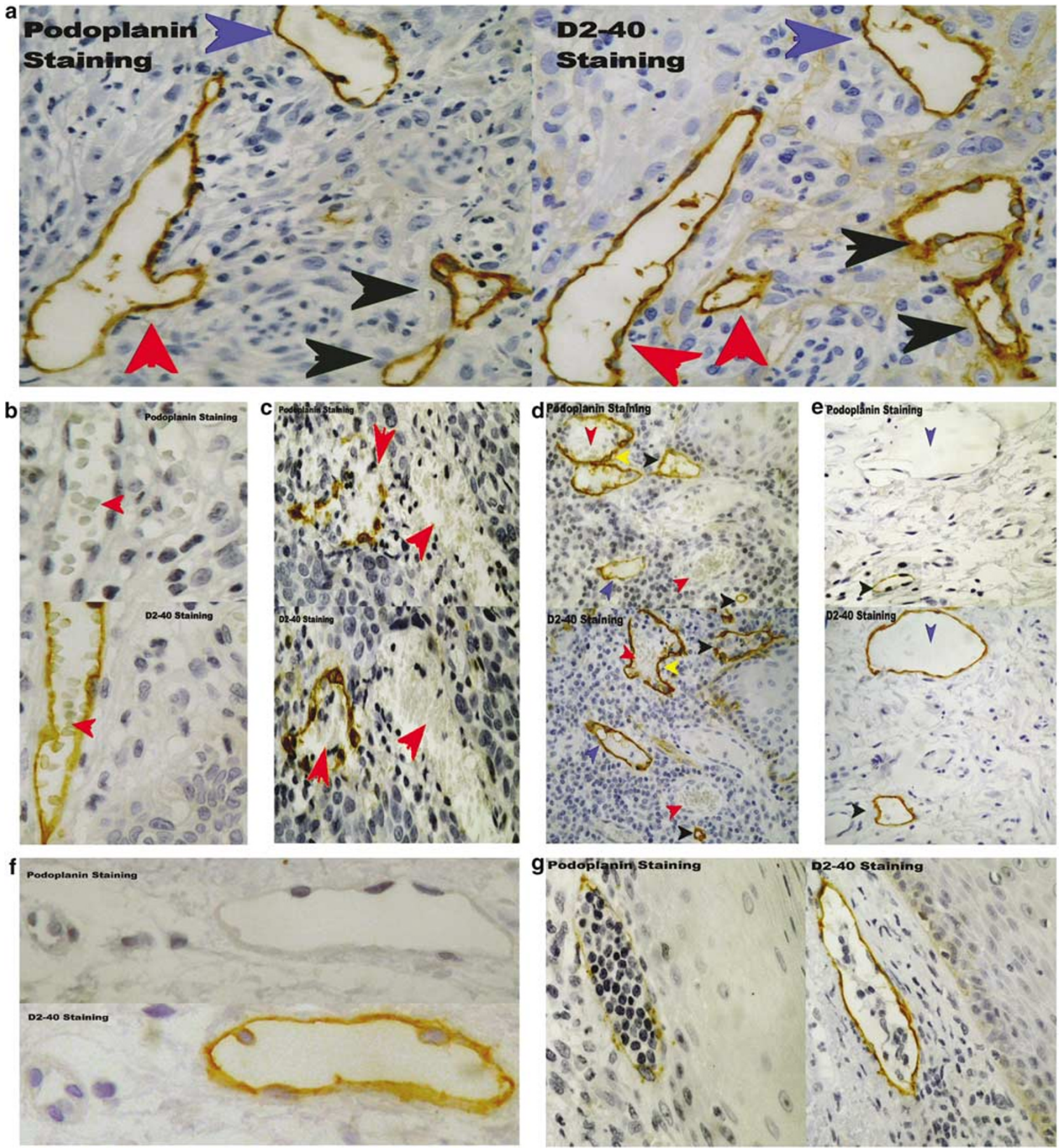

Figure 2 (a) Adjacent sections from the same tumor sample (laryngeal squamous cell carcinoma, SCC), in which the same vessels (indicated by arrows of the same color) are positively stained with both podoplanin and D2-40 (magnification $\times 400$ ). Vessels appearing joined in one section and separated in the other were counted as one, for both the sections. (b) Adjacent sections from the same tumor sample (laryngeal SCC), in which a vessel with luminal red blood cells (potential 'false-positive') is negative for podoplanin and positive for D2-40 (magnification $\times 1000$ ). Red arrows indicate red blood cells. (c) Adjacent sections from the same tumor sample (SCC of the floor of the mouth), in which a vessel with luminal red blood cells (potential 'false-positive') is positively stained with both podoplanin and D2-40 (magnification $\times 1000$ ). Notice the vessel close to the positive one, which has also luminal red blood cells (red arrow), but is negative for both the antibodies. (d) Adjacent sections from the same tumor sample (laryngeal SCC), in which both true- and falsepositive vessels can clearly be seen (magnification $\times 400$ ). All of these vessels are positive for both markers. Red arrows indicate red blood cells; arrows of the same color indicate the same vessels in the two sections. (e) Adjacent sections from the same tumor sample (SCC of the tongue), in which a vessel with luminal red blood cells (potential 'false-positive') is negative for podoplanin and positive for D2-40 (blue arrow, magnification $\times 4000$ ). There is also a vessel without red blood cells, which is positively stained with both markers (green arrow). (f) Adjacent sections from the same tumor sample (SCC of the lower lip), showing a vessel without luminal red blood cells, which is podoplanin negative and D2-40 positive (magnification $\times 1000$ ). (g) Adjacent sections from the same tumor sample (laryngeal SCC), showing a vessel filled with lymphocytes, without red blood cells, which is positively stained with both markers. 
Table 1 Counts of stained or unstained vessels with either antibody

\begin{tabular}{llrr}
\hline & & \multicolumn{2}{c}{$D 2-40$} \\
\cline { 3 - 4 } & & Stained & Unstained \\
\hline Podoplanin & Stained & 1864 & 119 \\
& Unstained & 391 & 21168
\end{tabular}
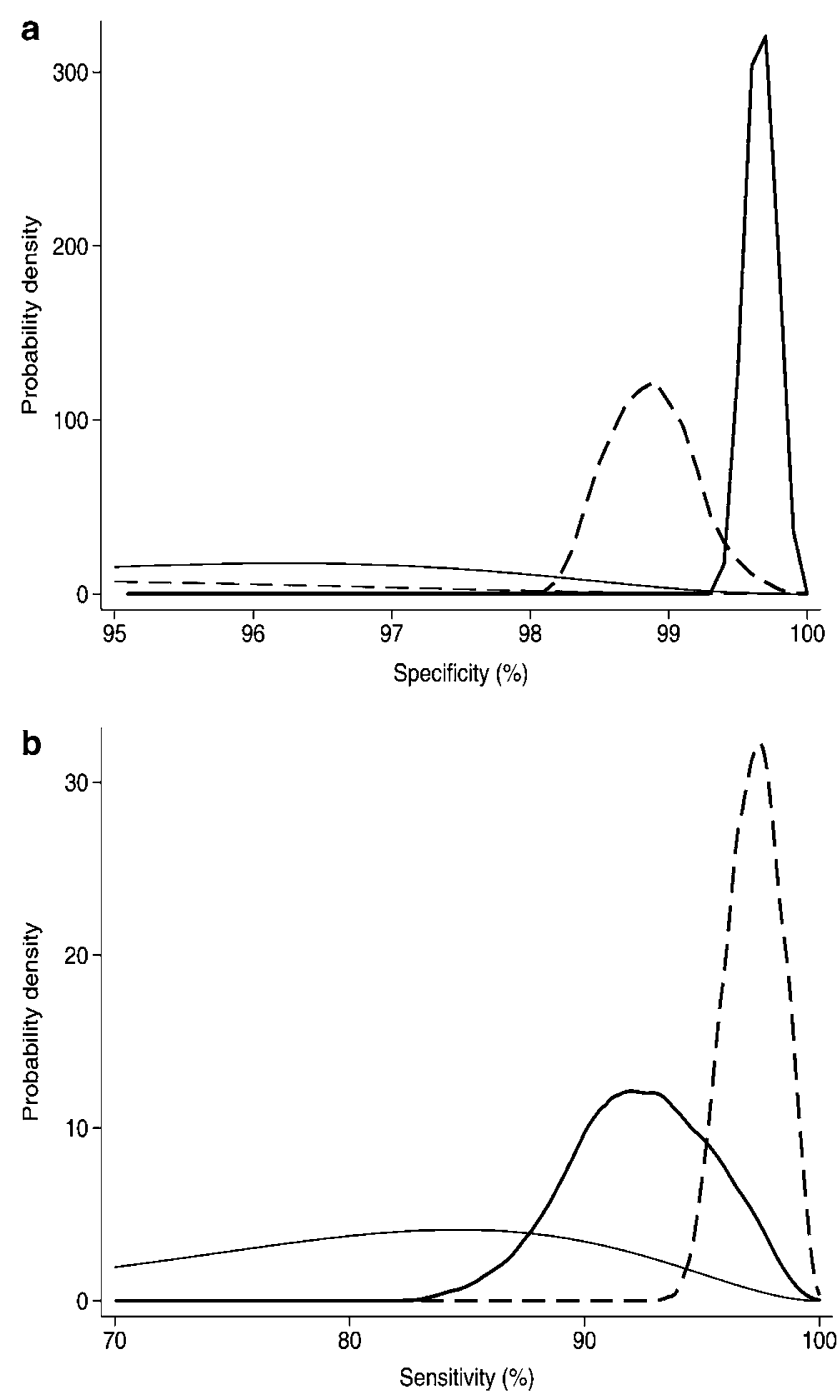

Figure 3 Posterior distributions assuming that the two antibodies do not yield statistically correlated results. The combination of the prior knowledge and the data yields the final estimates of the analysis (posterior knowledge). (a) Posterior distributions of the specificity of the antibody against podoplanin (thick solid line) and D2-40 (thick dashed line). For comparative reasons, the prior distributions for the specificities of the antibody agains podoplanin (thin solid line) and D2-40 (thin dashed line) are also shown. (b) Posterior distributions of the sensitivity of the antibody against podoplanin (thick solid line), and of D2-40 (thick dashed line). The thin solid line is the common prior for the sensitivities of the two antibodies.

D2-40 (97.3\% (95\% CrI: 94.9-99.2\%)) as shown in Figure 3b. Similar inferences were drawn when we used a different set of priors.
Table 2 Counts of vessels with intraluminal erythrocytes with either antibody

\begin{tabular}{llrr}
\hline & & \multicolumn{2}{c}{$D 2-40$} \\
\cline { 3 - 4 } & & Stained & Unstained \\
\hline \multirow{2}{*}{ Podoplanin } & Stained & 82 & 15 \\
& Unstained & 215 & 0 \\
\hline
\end{tabular}

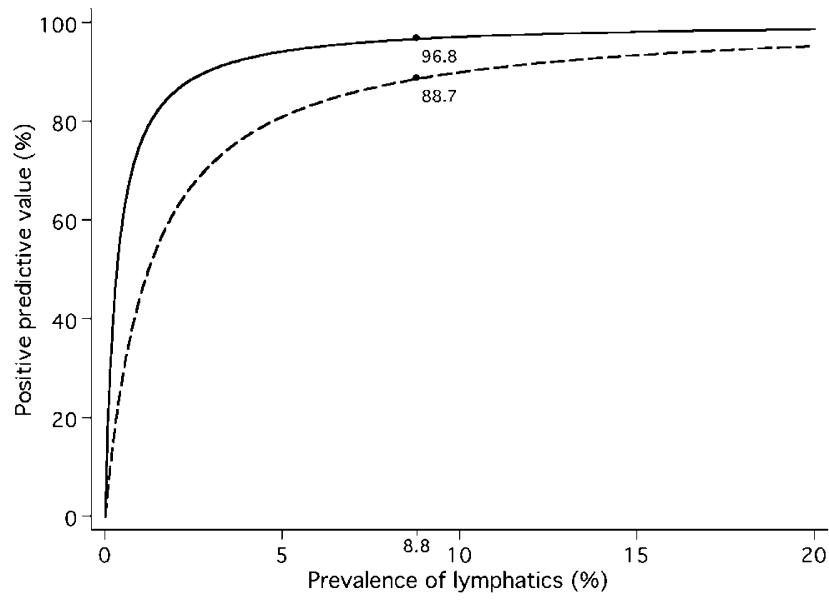

Figure 4 Positive predictive values for different prevalences of lymphatic vessels assuming that the two antibodies do not yield statistically correlated results. The proportion of the stained vessels that are truly lymphatic vessels (positive predictive value) for the antibody against podoplanin (solid line) and for D2-40 (dashed line) is plotted against the prevalence of the lymphatic vessels in a tissue section. When lymphatics are sparse, the difference in the positive predictive value increases. The dots represent the calculated values for our samples, where the estimated prevalence of the true lymphatics was $8.8 \%$.

The estimated prevalence of the lymphatic vessels was $8.8 \%$ (95\% CrI: $8.1-9.5 \%$ ). We calculated that the proportion of positively stained vessels (positive predictive values) that are truly lymphatics in our samples was $96.3 \%$ (95\% CrI: 94.2-98.6\%) and $88.9 \%$ (95\% CrI: $83.9-95.7 \%$ ) for podoplanin and D2-40, respectively. As evident from Figure 4, although the two antibodies had very similar diagnostic accuracy, the positive predictive values may differ, especially when lymphatics are sparse. Based on these estimates, the predicted proportion of falsely positively stained vessels would be 3.7 and $11.1 \%$ for the two antibodies, respectively. The corresponding observed proportions of stained vessels with intraluminal erythrocytes were 4.9 and $13.2 \%$ (Table 2).

The second set of analyses that allowed for correlation between the two tests yielded qualitatively similar results. Podoplanin and D2-40 were estimated to have specificities $95.6 \% \quad(95 \%$ CrI: 92.0-98.9\%) and $94.8 \%$ (95\% CrI: 90.9-98.3\%) and sensitivities $82.6 \% \quad(95 \%$ CrI: $62.7-94.7 \%)$ and 
90.0\% (95\% CrI: $74.0-97.6 \%$ ), respectively. The positive predictive value was $50.9 \%$ for podoplanin and $48.9 \%$ for D2-40.

\section{Discussion}

In the current study, we compare two lymphatic endothelium markers, and provide handy numerical estimates for their diagnostic accuracy. Our results suggest that both markers have extremely high specificity and sensitivity for the identification of lymphatic vessels, and have comparable positive predictive values in most settings.

Up to date, experts' opinions favored the antibody against podoplanin as the most reliable and specific marker at hand. ${ }^{1,5,9,14,15,17,21}$ Nevertheless, scientists emphasize the necessity for a quantitative evaluation of the accuracy of the so-called 'specific lymphatic endothelium markers'. ${ }^{1,5,19-22}$ To our knowledge, our work is the first report that pursues direct comparisons between two lymphatic endothelium markers and provides quantitative estimates for their specificity, sensitivity and positive predictive value. Contrary to prior beliefs, we found that both antibodies are essentially equally specific, in all sets of analyses. Thus, there may be little reason to prefer either one of the antibodies in most settings.

In our sections, $5 \%$ of the vessels stained by the antibody against podoplanin and $13 \%$ of those stained by D2-40 contained intraluminal red blood cells. These counts were very close to those calculated based on the positive predictive values of the two antibodies (approximately 4 and 11\%, respectively) under the assumption that the tests are statistically independent. This may be seen as an indirect support in favor of these findings, since the potential 'false-positive' counts were not taken into account in the Bayesian analyses. On the contrary, allowing for correlation between the two tests, one would calculate that almost half of the stained vessels were not lymphatics. Analyses allowing for correlation between the two antibodies are heavily dependent on the used prior distributions, and this might be a reasonable explanation for the high number of expected 'false-positives'. That high proportions of 'falsely positively' stained vessels would be incompatible with electron microscopic immunohistology findings ${ }^{26}$ where all examined podoplanin-stained vessels in normal skin were found to be lymphatics, and reciprocally, all lymphatics were stained with podoplanin. Taking all the above into account, we lend more credence to the inferences drawn from the model that assumes that the two tests are statistically independent.

The approach we used may yield reliable estimates for the prevalence ${ }^{30}$ of the lymphatic vessels in a tissue section. One of the main drawbacks of the assessment of the lymphatic vessel density in histological sections is the very high interobserver variability in the identification of the areas with the highest vessel concentration, the so-called 'hotspots'. ${ }^{34}$ On the contrary, the prevalence of the lymphatics in a section is not so subjective, since the assessor simply counts everything. These prevalence estimates might be equally good or better predictors of clinical outcomes compared to the assessment of the lymphatic vessel density. Moreover, the whole procedure could be automated through specialized software, using a PC connected to a light microscope. ${ }^{35}$

Some limitations of our study should be discussed. First, we estimated the total number of vessels (blood and lymphatics) using CD34. It has been reported that CD34 is expressed both in blood vessels and lymphatics, but blood vessels stain more intensely. ${ }^{24,25,36}$ Thus, the total number of vessels we counted may be an underestimation of the true value. However, this did not bias our findings, as evident from sensitivity analyses. Second, we did not perform double immunostaining, as this would be technically more difficult. Nevertheless, given our elaborate methodology for the evaluation of the staining patterns, large misclassification is unlikely. Third, there is an inherent subjectivity in Bayesian approaches, expressed by the morphology of the prior distributions. The choice of the prior distributions mostly affects inferences based on the assumption that the two antibodies are statistically dependent. However, the used priors are based on the available knowledge and a comprehensive literature search. ${ }^{1,5,9,14,15,17,21,26}$ Finally, we considered stained vessels with intraluminal red blood cells as potential 'false-positives'. Intraluminal erythrocytes might be a histologic artifact resulting from pressure-induced leakage of a neighboring blood vessel in a true lymphatic vessel. ${ }^{26}$ This phenomenon is observed in lymphangiomas and might also happen in rapidly growing tissues, like malignant tumors. ${ }^{26}$ However, we believe that the majority of the potential 'false-positives' are indeed blood vessels; otherwise, the two antibodies ought to yield roughly the same number of potential 'falsepositive' vessels.

Allowing for these caveats, both antibodies can be regarded as reference standards for the identification of lymphatic vessels in most settings. Similar approaches could be employed for the evaluation of diagnostic accuracy of other markers, such as the remaining lymphatic endothelium markers, antibodies detecting blood vessels or antibodies from different manufacturers detecting the same antigen.

\section{Acknowledgement}

We thank Silvana Geleff for kindly providing us the antibody against podoplanin, and for her useful comments. 


\section{References}

1 Saharinen P, Tammela T, Karkkainen MJ, et al. Lymphatic vasculature: development, molecular regulation and role in tumor metastasis and inflammation. Trends Immunol 2004;25:387-395.

2 Padera TP, Kadambi A, di Tomaso E, et al. Lymphatic metastasis in the absence of functional intratumor lymphatics. Science 2002;296:1883-1886 (Epub 2002 April 25).

3 Reis-Filho JS, Schmitt FC. Lymphangiogenesis in tumors: what do we know? Microsc Res Technol 2003; 60:171-180.

4 Jain RK, Fenton BT. Intratumoral lymphatic vessels: a case of mistaken identity or malfunction? J Natl Cancer Inst 2002;94:417-421.

5 Stacker SA, Achen MG, Jussila L, et al. Lymphangiogenesis and cancer metastasis. Nat Rev Cancer 2002;2: 573-583.

6 Beasley NJ, Prevo R, Banerji S, et al. Intratumoral lymphangiogenesis and lymph node metastasis in head and neck cancer. Cancer Res 2002;62:1315-1320.

7 Straume O, Jackson DG, Akslen LA. Independent prognostic impact of lymphatic vessel density and presence of low-grade lymphangiogenesis in cutaneous melanoma. Clin Cancer Res 2003;9:250-256.

8 Schoppmann SF, Bayer G, Aumayr K, et al. Austrian Breast and Colorectal Cancer Study Group. Prognostic value of lymphangiogenesis and lymphovascular invasion in invasive breast cancer. Ann Surg 2004;240: 306-312.

9 Munoz-Guerra MF, Marazuela EG, Martin-Villar E, et al. Prognostic significance of intratumoral lymphangiogenesis in squamous cell carcinoma of the oral cavity. Cancer 2004;100:553-560.

10 Maula SM, Luukkaa M, Grenman R, et al. Intratumoral lymphatics are essential for the metastatic spread and prognosis in squamous cell carcinomas of the head and neck region. Cancer Res 2003;63:1920-1926.

11 Laura R-B, Benoit T, Emile G, et al. Lymphatic vessel density and vascular endothelial growth factor-C expression correlate with malignant behavior in human pancreatic endocrine tumors. Clin Cancer Res 2004;10:6919-6928.

12 Franchi A, Gallo O, Massi D, et al. Tumor lymphangiogenesis in head and neck squamous cell carcinoma: a morphometric study with clinical correlations. Cancer 2004;101:973-978.

13 Kyzas PA, Geleff S, Batistatou A, et al. Lymphangiogenesis in head and neck squamous cell carcinoma: Evidence and prognostic implications. J Pathol 2005; 206:170-177.

14 Matsui K, Nagy-Bojarsky K, Laakkonen $\mathrm{P}$, et al. Lymphatic microvessels in the rat remnant kidney model of renal fibrosis: aminopeptidase $\mathrm{p}$ and podoplanin are discriminatory markers for endothelial cells of blood and lymphatic vessels. J Am Soc Nephrol 2003;14:1981-1989 (Erratum in J Am Soc Nephrol 2003; 14(10): following table of contents).

15 Birner P, Obermair A, Schindl M, et al. Selective immunohistochemical staining of blood and lymphatic vessels reveals independent prognostic influence of blood and lymphatic vessel invasion in early-stage cervical cancer. Clin Cancer Res 2001;7:93-97.

16 Valencak J, Heere-Ress E, Kopp T, et al. Selective immunohistochemical staining shows significant prognostic influence of lymphatic and blood vessels in patients with malignant melanoma. Eur J Cancer 2004;40:358-364.

17 Clarijs R, Ruiter DJ, de Waal RM. Lymphangiogenesis in malignant tumours: does it occur? J Pathol 2001; 193:143-146.

18 Dumoff KL, Chu C, Xu X, et al. Low D2-40 immunoreactivity correlates with lymphatic invasion and nodal metastasis in early-stage squamous cell carcinoma of the uterine cervix. Mod Pathol 2005;18: 97-104.

19 Chu AY, Litzky LA, Pasha TL, et al. Utility of D2-40, a novel mesothelial marker, in the diagnosis of malignant mesothelioma. Mod Pathol 2005;18: 105-110.

20 Choi WW, Lewis MM, Lawson D, et al. Angiogenic and lymphangiogenic microvessel density in breast carcinoma: correlation with clinicopathologic parameters and VEGF-family gene expression. Mod Pathol 2005; 18:143-152.

21 Cunnick GH, Jiang WG, Gomez KF, et al. Lymphangiogenesis and breast cancer metastasis. Histol Histopathol 2002;17:863-870.

22 Pepper MS. Lymphangiogenesis and tumor metastasis: myth or reality? Clin Cancer Res 2001;7:462-468.

23 Kahn HJ, Marks A. A new monoclonal antibody, D2-40, for detection of lymphatic invasion in primary tumors. Lab Invest 2002;82:1255-1257.

24 Tsuji T, Sasaki Y, Tanaka M, et al. Microvessel morphology and vascular endothelial growth factor expression in human colonic carcinoma with or without metastasis. Lab Invest 2002;82:555-562.

25 Saad RS, Liu YL, Nathan G, et al. Endoglin (CD105) and vascular endothelial growth factor as prognostic markers in colorectal cancer. Mod Pathol 2004;17: 197-203.

26 Breiteneder-Geleff S, Soleiman A, Kowalski H, et al. Angiosarcomas express mixed endothelial phenotypes of blood and lymphatic capillaries: podoplanin as a specific marker for lymphatic endothelium. Am J Pathol 1999;154:385-394.

27 Breiteneder-Geleff S, Matsui K, Soleiman A, et al. Podoplanin, novel 43-kd membrane protein of glomerular epithelial cells, is down-regulated in puromycin nephrosis. Am J Pathol 1997;151:1141-1152.

28 Karkkainen MJ, Makinen T, Alitalo K. Lymphatic endothelium: a new frontier of metastasis research. Nat Cell Biol 2002;4:2-5.

29 Loong TW. Understanding sensitivity and specificity with the right side of the brain. BMJ 2003;327: 716-719.

30 Joseph L, Gyorkos TW, Coupal L. Bayesian estimation of disease prevalence and the parameters of diagnostic tests in the absence of a gold standard. Am J Epidemiol 1995;141:263-272.

31 Schacht V, Dadras SS, Johnson LA, et al. Up-regulation of the lymphatic marker podoplanin, a mucin-type transmembrane glycoprotein, in human squamous cell carcinomas and germ cell tumors. Am J Pathol 2005;166:913-921.

32 Georgiadis MP, Johnson WO, Gardner IA, et al. Correlation-adjusted estimation of sensitivity and specificity of two diagnostic tests. J R Stat Soc Ser C 2003;52:63-76.

33 Dendukuri N, Lawrence J. Bayesian approaches to modeling the conditional dependence between multiple diagnostic tests. Biometrics 2001;57:158167. 
34 Vermeulen PB, Gasparini G, Fox SB, et al. Second international consensus on the methodology and criteria of evaluation of angiogenesis quantification in solid human tumours. Eur J Cancer 2002;38: 1564-1579.

35 Hannen EJ, van der Laak JA, Manni JJ, et al. Computer assisted analysis of the microvasculature in metastasized and nonmetastasized squamous cell carcinomas of the tongue. Head Neck 2002;24: $643-650$.

36 Schmid K, Birner P, Gravenhorst V, et al. Prognostic value of lymphatic and blood vessel invasion in neuroendocrine tumors of the lung. Am J Surg Pathol 2005;29:324-328.

Supplementary Information accompanies the paper on Modern Pathology website (http://www.nature.com/ modpathol) 